Marquette University

e-Publications@Marquette

2013

\title{
Time Resolved Gain and Excess Noise Properties of InGaAs/ InAIAs Avalanche Photodiodes with Cascaded Discrete Gain Layer Multiplication Regions
}

Georges M. Williams

Voxtel Inc.

David A. Ramirez

University of New Mexico

Majeed M. Hayat

Marquette University, majeed.hayat@marquette.edu

Andrew S. Huntington

Voxtel, Inc.

Follow this and additional works at: https://epublications.marquette.edu/electric_fac

Part of the Computer Engineering Commons, and the Electrical and Computer Engineering Commons

\section{Recommended Citation}

Williams, Georges M.; Ramirez, David A.; Hayat, Majeed M.; and Huntington, Andrew S., "Time Resolved Gain and Excess Noise Properties of InGaAs/InAIAs Avalanche Photodiodes with Cascaded Discrete Gain Layer Multiplication Regions" (2013). Electrical and Computer Engineering Faculty Research and Publications. 567.

https://epublications.marquette.edu/electric_fac/567 
Marquette University

e-Publications@Marquette

\section{Electrical and Computer Engineering Faculty Research and Publications/College of Engineering}

This paper is NOT THE PUBLISHED VERSION; but the author's final, peer-reviewed manuscript. The published version may be accessed by following the link in th citation below.

Journal of Applied Physics, Vol. 113, No. 9 (2013). DOI. This article is (C AIP Publishing and permission has been granted for this version to appear in e-Publications@Marquette. AIP Publishing does not grant permission for this article to be further copied/distributed or hosted elsewhere without the express permission from AIP Publishing.

\section{Time Resolved Gain and Excess Noise Properties of InGaAs/InAIAs Avalanche Photodiodes with Cascaded Discrete Gain Layer Multiplication Regions}

George M. Williams

Voxtel Inc., 15985 NW Schendel Ave., Beaverton, Oregon

David A. Ramirez

Center for High Technology Materials, University of New Mexico, Albuquerque, New Mexico

Majeed M. Hayat

Center for High Technology Materials, University of New Mexico, Albuquerque, New Mexico Andrew S. Huntington

Voxtel Inc., 15985 NW Schendel Ave., Beaverton, Oregon 


\section{ABSTRACT}

To predict pulse detection performance when implemented in high speed photoreceivers, temporally resolved measurements of a 10-stage InAIAs/InGaAs single carrier multiplication (SCM) avalanche photodiode (APD)'s avalanche response to short multi-photon laser pulses were explained using instantaneous (time resolved) pulse height statistics of the device's impulse response. Numeric models of the junction carrier populations as a function of the time following injection of a primary photo-electron were used to create the probability density functions (pdfs) of the instances of the avalanche buildup process. The numeric pdfs were used to generate low frequency gain and excess noise models, which were in good agreement with analytic models of multiple discrete low-gain-stage APDs and with measured excess noise data. The numeric models were then used to generate the instantaneous and cumulative instantaneous low order statistics of the instances of the impulse response. It is shown that during the early times of the impulse response, the SCM APDs have lower excess noise than the pseudo-DC measurements and the common APDmodels used to describe them. The methods of determining the time resolved low order statistics of APDs are described and the importance of using time-resolved models of APDgain and noise is discussed.

\section{INTRODUCTION}

Voxtel has developed a single carrier multiplication (SCM) avalanche photodiode (APD) in the $\mathrm{In}_{0.52} \mathrm{Al}_{0.48} \mathrm{As} / \mathrm{In}_{0.53} \mathrm{GaO}_{.47} \mathrm{As}$ material system, which is epitaxial grown, lattice-matched to $\mathrm{n}$-type InP substrates, by molecular beam epitaxy (MBE). Like other back-illuminated InGaAs detectors, the SCM APD is sensitive in the short-wavelength infrared (SWIR) from 950 to $1700 \mathrm{~nm}$. Structurally, the SCM APD, shown in Figure $\underline{1}$, is a separate absorption-charge-multiplication (SACM) device with a 1.5- $\mu$ m-thick InGaAs absorber and a multiplication region that consists of multiple cascaded $p-i-p-i-n-i \operatorname{InAIAs} / \operatorname{InAIGaAs}$ gain stages, each about $200 \mathrm{~nm}$ thick. The doping pattern and alloy selection within each gain stage are designed to modulate carrier energy and impact-ionization threshold so as to favor electron-initiated impact-ionization over hole ionization and restrict carrier multiplication to a single 30-nm layer in each gain stage. SCM APDs with 5, 7, and 10 gain-stages have been produced. The impulse response statistics of the $\mathrm{J}=10$ stage devices are reported here. 


\begin{tabular}{|c|c|c|}
\hline & & \\
\hline & $150 A \operatorname{InG} A$ As $\left(4 \times 10^{19} \mathrm{~cm}^{-3} \mathrm{p}\right)$ & \\
\hline & 400 A grade $\left(10^{19} \mathrm{~cm}^{-3} \mathrm{p}\right)$ & \\
\hline & $4050 \mathrm{~A}$ InAlAs $\left(10^{19} \mathrm{~cm}^{-3} \mathrm{p}\right)$ & anode \\
\hline & $400 \mathrm{~A}$ grade $\left(5 \times 10^{18} \mathrm{~cm}^{-3} \mathrm{p}\right)$ & \\
\hline & $400 \mathrm{~A} \operatorname{InG} \mathrm{AAs}\left(10^{10} \mathrm{~cm}^{-3} \mathrm{p}\right)$ & \\
\hline & $1.46 \mu \mathrm{m}$ InGaAs (i) & absorber \\
\hline & 400 A grade (i) & \\
\hline & $250 \AA$ InAlAs $\left(10^{18} \mathrm{~cm}^{-3} \mathrm{p}\right)$ & charge layer \\
\hline & 350 A InAlAs (i) & \\
\hline & $100 \mathrm{~A}$ InAlAs $\left(10^{18} \mathrm{~cm}^{-3} \mathrm{p}\right)$ & field up \\
\hline & $50 \mathrm{~A}$ InAIAS (i) & \\
\hline & $200 \mathrm{~A} 70 \%$ InAlAs $/ 30 \%$ InGaAs (i) & ionization \\
\hline & So A InAlAs (i) & \\
\hline & 160 A InAIAs $\left(7.0 \times 10^{17} \mathrm{~cm}^{3} \mathrm{n}\right)$ & field down \\
\hline & $1000 \mathrm{~A}$ InAlas (i) & relaxation \\
\hline & 100 A InALAs $\left(10^{18} \mathrm{~cm}^{13} \mathrm{p}\right)$ & field up \\
\hline & 300 A InAlAs (i) & \\
\hline & $100 \mathrm{~A} \operatorname{InAlAS}\left(10^{18} \mathrm{~cm}^{.3} \mathrm{p}\right)$ & field up \\
\hline & SOA InAlAs (i) & \\
\hline & 200 A $70 \%$ InAlAs / $30 \%$ InGaAs (i) & ionization \\
\hline & $50 \mathrm{~A}$ InAlAs (i) & \\
\hline & $1000 \mathrm{~A}$ InAlAs $\left(1 \times 10^{13} \mathrm{~cm}^{-3} \mathrm{n}\right)$ & \\
\hline & 1840 A InAlAs $\left(8 \times 10^{18} \mathrm{~cm}^{-3} \mathrm{n}\right)$ & \\
\hline & InP substrate (n) & \\
\hline
\end{tabular}

FIG. 1.Epitaxial layer structure of an SCM APD with $n+1$ stages.

In Figure $\underline{2}$ is plotted the output from a Monte Carlo simulation of the spatial distribution of impact-ionization inside a 10-gain-stage multiplier, overlaid on the SCM APD's axial electric field profile. Primary photoelectrons are injected from the left and multiply sequentially in each of the 10 gain stages as they drift to the right. The asymmetric doping and alloy composition pattern of each gain stage are ordered such that electrons are preheated prior to injection into a layer with a low impact-ionization threshold, whereas the counter-propagating holes encounter the same regions in opposite order, and are injected into the multiplication layer cold. The resulting enhancement of the electron-initiated impact-ionization rate and suppression of hole ionization increases the electron ionizationprobability, $P_{e}$, and suppresses the hole ionization probability, $P_{h}$, in each gain stage, so that the gain is more deterministic than would occur in a homogenous multiplier.

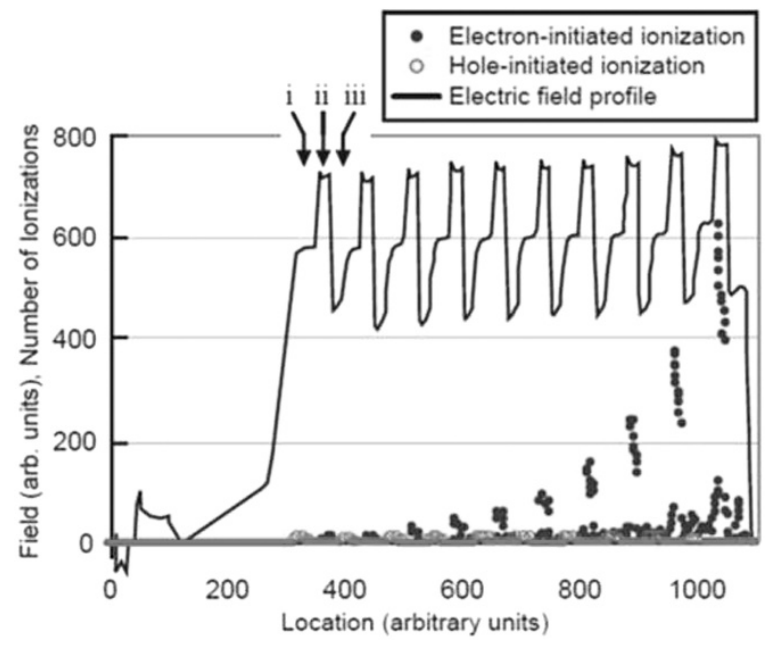


FIG. 2.A plot of the spatial count of hole- and electron-initiated impact ionization events generated by a 1D Monte Carlo simulation superimposed on the electric field profile of the 10-gain-stage SCM APD architecture multiplier. The absorption layer (to the left of the diagram) is not shown.

The effect of the SCM APD device structure and materials on the performance of a photoreceiver requires that a complete statistical description of the charge induced in the receiver circuits by the motion of the carriers in the junction be modeled on the time scales of the optical input pulses and integration times of the receiver circuits. The introduction of time dynamics into the calculations has no effect on the statistical moments or on the counting distribution, as long as the integration time is sufficiently long so that the entire current pulse is captured as a charge. However, when circuit decisions are based on photocurrent integrated over shorter times than the impulse response, such is common in digital information systems, new approaches to predicting receiver performance are required.

\section{DC EXCESS NOISE MODELS}

The excess noise factor of a linear-mode APD is normally defined in the low-frequency (quasi-DC) limit, meaning that the gain statistics are treated assuming sufficient time for all impact-ionization chains generating secondary carriers to complete before the end of the signal pulse. The avalanche noise in an APD is generally characterized by the normalized second central moment of the gain random variable for a single input photocarrier:

$$
F\left(M_{D C}\right)=\left\langle M_{D C}{ }^{2}\right\rangle /\left\langle M_{D C}\right\rangle^{2}
$$

The pseudo-DC excess noise factor is most often indirectly calculated from the measurednoise power, photocurrent, and gain using the assumption

$$
F\left(M_{D C}\right)=S_{I}(0) /\left(2 q\left\langle M_{D C}\right\rangle^{2} I_{0}\right),{ }^{(2)}
$$

where $S_{1}(0)$ is the noise power at $0 \mathrm{~Hz}$ and $I_{0}$ is the unity-gain photocurrent. The current power can be obtained using the Wiener-Khinchin theorem by Fourier transform of the current autocorrelation function, $\underline{1}$ i.e.

$$
S_{I}(0)=2 q^{2} \operatorname{var}(n)=2 \mathrm{q}^{2} \quad\langle\mathrm{n}\rangle=2 \mathrm{q}\langle\mathrm{l}\rangle
$$

The DC component of $I(\mathrm{t})$, after autocorrelation and Fourier transform, becomes the signal power, $\langle l\rangle{ }^{2} \delta(\mathrm{f})$, and it can be shown that at $f \sim 0 \mathrm{~Hz}, \underline{1}$

$$
S_{I}(0)=2 q^{2} \operatorname{var}(n)=2 \mathrm{q}^{2} \quad\langle\mathrm{n}\rangle=2 \mathrm{q}\langle\mathrm{l}\rangle ;
$$

this is the Schottky theorem for shot noise. Assuming $n$ electrons are initially photo-generated and undergo avalanche multiplication, each with a multiplication of $M_{i}$, under the assumptions that the photogenerated electrons are independent of each other, i.e., a Poisson distribution, and $\left\langle M_{i}\right\rangle=\langle M\rangle$ and $\left\langle M_{i}{ }^{2}\right.$ \rangle$=\left\langle M^{2}\right\rangle$, the Burgess variance theorem ${ }^{2}$ can be applied so that

$$
S_{I}(0)=2 q\left(\left\langle M_{D C}^{2}\right\rangle /\left\langle M_{D C}\right\rangle^{2}\right)\left\langle M_{D C}\right\rangle^{2} I_{0}=2 q F\left(\left\langle M_{D C}\right\rangle\right)\left\langle M_{D C}\right\rangle^{2} I_{0},
$$


where $F\left(M_{D C}\right)$ is the excess noise factor that describes the amount the signal's noise is degraded from its lower (shot noise) limit. The validity of $\underline{(5)}$ depends critically on the assumption that the multiplied current pulses resulting from each photoelectron have negligible width, i.e., "instantaneous amplification."

Traditional Mclntyre APD theory assumes DC operation and bases excess noise predictions on a uniform avalanche medium and an impact-ionization process independent of carrier history $\mathbf{4}^{4}$

$$
F\left(M_{D C}, k\right)=M_{D C}\left[1-(1-k)\left(\frac{M_{D C}-1}{M_{D C}}\right)^{2}\right] .
$$

Conventionally, $k$ defines the ratio of the hole-initiated ionization rate to the electroninitiated ionization rate, $k=6 / \alpha$. Although in practice, we often use the inverse ratio $1 / k$ to facilitate comparisons between materials, and for heterostructured APDs, we often use an empirically found effective ionization ratio, $k_{\text {eff, }}$ which includes both the device structure effects and the inherent semiconductor material properties. Hereafter, we refer to $k, 1 / k$, and $k_{\text {eff }}$ simply as $k$, such that parameterized by a low $k,(6)$ results in a gain distribution more tightly distributed around $M_{D c}$; whereas with higher $k$ values, the gain of individual events is much more widely distributed about the mean, and the APD response is less correlated to the optical input. $\frac{5}{5}$

Because they represent, in a compact way, the lowest order statistical properties of the DC gain fluctuations that impose multiplicative noise on individual photon detections (excess noise), the McIntyre equation (6) and the electron count variance are useful statistics. However, Mclntyre analysis assumes that impact ionization is a continuous local process, and that the ionization probability is only a function of the local electric field, irrespective of a carrier's ionization history; it is correct only at high gains and when the pulse width is longer than the duration of the impulse response.., 5 For these reasons, the Mclntyre formula does not necessarily apply to the SCM APD.

As the SCM APD is designed with a multiplication region comprised multiple discrete gain layers, when the number of ionizing collisions per primary transit is low, the discrete nature of the avalanche gain process must be taken into account. Such processes have been analyzed by Lukaszek et al. $\underline{\underline{6}}$ and by Van Vliet and Rucker, $\underline{\underline{7}}$ who predict a lowering of the excess noise factor compared to McIntyre predictions. Although their treatment was intended to describe multiplication noise in conventional APD's, their use of a deterministic number of shiftedBernoulli stages makes their model suitable for the instantaneous-multiplication discrete multi-stage device.

Although structurally different, the gain and noise characteristics of an ideal multi-discrete-gain-stage SCM APD with no hole ionization feedback (i.e., $k=0$ ) can be described using the approached developed by Capasso et al. $.9,9$ for a "staircase" solid-state photomultiplier. For a multi-stage multiplier comprised discrete gain regions, if a secondary electron-hole pair is generated for each electron injected into a given gain stage and there is zero hole-initiated multiplication, the end-to-end gain for a photoelectron injected into the cascaded multiplier is $M_{D C}=2^{J}$, where $J$ is the total number of gain stages. In this ideal case, the multiplication is deterministic and the avalanche gain is a constant.

In practice, the avalanche gain per stage is less than 2 because the probability of electronionization, $P_{e}$, is less than one, and for $0 \leq P_{e}<1$, the probability of the random variable that an electron injected into a gain stage fails to impact-ionize is $\delta=1-P_{e}$, where $\delta$ is the fraction of electrons that do not impact ionize. Assuming uniform properties across all gain stages, the average end-to-end gain is then determined by 


$$
M_{D C}=(2-\delta)^{J}
$$

and the excess noise by $\frac{7,8,10}{}$

$$
F(J, \delta)=1+\frac{\delta\left[1-(2-\delta)^{-J}\right]}{(2-\delta)}
$$

Note that in $(8) F(J, \delta)$ is $<2$ for any $J$, and when $P_{e}=1$, in the absence of carrier fluctuations, $\operatorname{var}(J, \delta)=\delta(1-\delta)=0$ and $F\left(M_{D C}\right)=1$, whereas for $(6)$, the McIntyre model parameterized by $k=0$ approaches $F\left(M_{D C}\right)=2$ at high gain.

In practice, SCM APDs also may accumulate gain through hole initiated ionization. To accommodate both electron and hole ionization in a multi-stage discretized APD, the DC gain can be approximated by Van Vliet et al. 11

$$
M_{D C}(J)=\frac{\left(1+P_{e}\right)^{J}\left(1-k_{s}\right)}{\left(1+k_{s} P_{e}\right)^{J+1}-k_{s}\left(1+P_{e}\right)^{J+1}}
$$

$$
F\left(M_{D C}, k_{s}, P_{e}\right)=1+\frac{\left(1-\frac{1}{M_{D C}}\right)\left(1-k_{s}\right)}{2+P_{e}\left(1-k_{s}\right)} *\left[-P_{e}+2 \frac{\left(1-k_{s} P_{e}^{2}\right)}{\left(1+k_{s} P_{e}\right)}\left(M_{D C} k_{s} \frac{\left(1+P_{e}\right)}{\left(1-k_{s}\right)}+\frac{1}{1+P_{e}}\right)\right] .
$$

Here, $k_{s}=P_{h} / P_{e}$ defines the ratio of the hole-ionization probability per stage, $P_{h}$, to the electron-ionization probability per stage $P_{e}$, where the distributed ionization coefficients $\alpha$ and $B$ are lumped into the probabilities $P_{e}$ and $P_{h}$, respectively; $P_{h}=e^{b d}-1$ and $P_{e}=e^{\alpha d}-1$, where $d$ is the length of each individual gain stage, and in the limit, $k_{s} \approx b / \alpha$.

Shown in Figure $\underline{3}$ is a plot of the measured $F\left(M_{D C}\right)$ values for a 10-stage SCM APD, plotted against the excess noise predicted by the Monte Carlo simulations shown in Figure $\underline{2}$. The Monte Carlo simulated and measured excess noise data show good agreement with each other and with (10) parameterized by $k_{s}=0.036$. Shown for comparison is a plot of the McIntyre equation (6) parameterized by $k=0.036$. As expected, the SCM APD excess noise is lower than that predicted by $(6)$ for most bias conditions. Only at high bias conditions (i.e., above about $\left.\left\langle M_{D C}\right\rangle=150\right)$, does the Mclntyre model approach the SCM APD measuredexcess noise data and the excess noise approximated by $\left(10 ; k_{s}=0.036\right)$. From $(9)$, a mean gain of $\left\langle M_{D C}\right\rangle=150$ requires that $P_{e}=0.355$, and with $k_{s}=0.036, P_{h}=0.0128$. The lower excess noise of the SCM APD, compared to bulk semiconductor multiplication APDsdescribed by $(6)$, is due to fact that carrier ionization occurs only at well-defined positions in space (at the low threshold regions of the gain stages), a significant portion of electroncarriers ionize at each gain stage, and hole ionization is low; thus the statistical variations of the gain are small. 


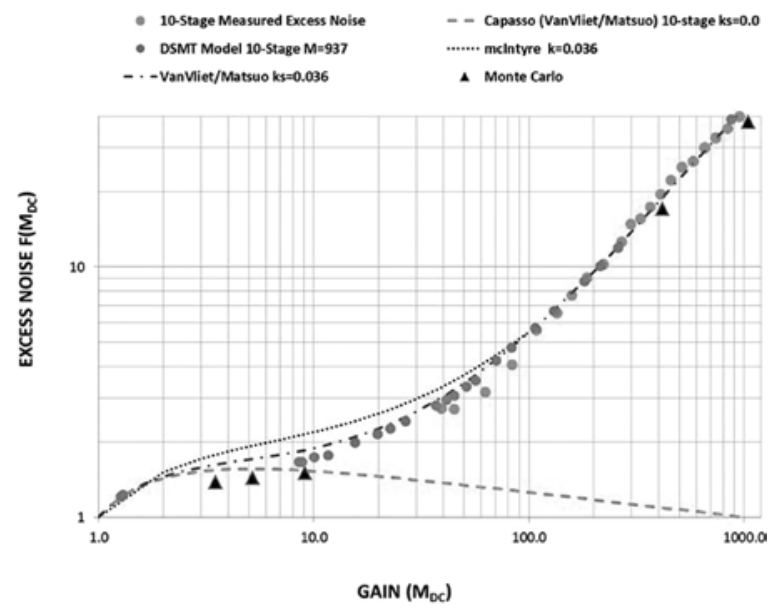

FIG. 3.Plot of the data from the numeric "scattering aware" DSMT model of the SCM APD compared to the Monte Carlo model data used to design the 10-stage SCM APD, measured data, and the analytical model of VanVliet (8), showing both $\mathrm{k}_{\mathrm{s}}=0.036$ and $\mathrm{k}_{\mathrm{s}}=0$.

Having verified the numerical model useful for describing the SCM APD's pseudo-DC response to optical pulses longer than the device's impulse response, next a predictive model of the instantaneous excess noise for the earliest times of the impulse response to short optical pulses was sought.

\section{SCM APD IMPULSE RESPONSE PROPERTIES}

The pseudo-DC measures of avalanche noise, as they assume that the optical pulse is longer than the APD's impulse response, are without reference to the temporal dynamics of the impact-ionization process. Analysis of the role that the underlying device structure and semiconductor materials play in the carrier dynamics allows us to obtain a better understanding of the complex interplay between the avalanche buildup time and the partial gain that determine the instantaneous statistics of the electrical current induced in the external decision circuits by motion of the carriers in the device.

The current pulse arising from single photon absorption can be modeled as a superposition of contributions from the initial photoelectron and its subsequent generations of ionizing charge carrier progeny. The physical basis of the impulse response function is most readily understood in terms of the gain stage transit times of the two carrier species. Using an electron velocity $v_{e}$ and a hole velocity $v_{h}$, the transit times of the two carrier species across a gain stage layer of length $d$ are $s_{e}$ and $s_{h}$, respectively. As the net velocity of electronsgenerally typically from that of holes, we can define $r=s_{e} / s_{h}$. Subsequently, the gain stage transit time is permitted to assume the form of a random variable. Although the probability density functions (pdfs) of the transit time can differ from stage to stage, it is reasonable to choose them to be independent identically distributed random variables. $\underline{13}$ Assuming, for the sake of simplicity, that $s=s_{e}=s_{h}$, so that $v=v_{e}=v_{h}$, and $r=1$, the transit time of the multiplication region can be defined at deterministic transit time $T=J \tau$, which is the time it takes the carriers to transit the length of the multiplication region, $D$, where $D=J d$.

The SCM APDs InAIAs multiplication region is $2 \mu \mathrm{m}$ thick, and assuming equal carrier velocities of $v=9.31 \times 10^{5} \mathrm{~cm} \mathrm{~s}^{-1}$, the transit time of the multiplication region is about $T=276 \mathrm{ps}$. The SCM APD includes a $1.5 \mu \mathrm{m}$ thick InGaAs absorption layer, so the total junction length is $L=3.5 \mu \mathrm{m}$, and the total junction transit time is $1.75 T$, or about 500 ps.

The total current delivered to an external circuit by the carriers in the junction of the APDcan be calculated using Ramo's theorem $\underline{14}$ 


$$
i_{T}(t)=\left(q /(L) \sum v_{e}(t)+(q / L) \sum v_{h}(t)\right.
$$

where the sums are taken over all of the carriers present in the junction at one time. Therefore, the instantaneous current contribution of a carrier is $v q L^{-1}$, and the properties of the instantaneous current can be analyzed by tracking the populations of hole and electroncarriers over all of the times of the impulse response. $\underline{15}$

To describe the excess noise at the instances of the SCM APD's impulse response, it is helpful to define a mean instantaneous gain, $\left\langle M\left(t, M_{D C}\right)\right\rangle$, and an instantaneous excess noise factor, $F\left(\left\langle M\left(t, M_{D C}\right)\right\rangle\right)$, which for an APD biased for $\left\langle M_{D C}\right\rangle$, describe the contributions to the gain and excess noise, respectively, by carriers present in the junction at any instance, $t$, of the impulse response from a single photon. The cumulative instantaneous excess noise $F\left(M_{D C}(\tau)\right)$ is the excess noise integrated during the accumulation of the partial gain, $M_{D C}(\tau)$, where $M_{D C}(\tau)=\int_{t=0}^{\tau} M\left(t, M_{D C}\right) d t$.

The temporal dynamics of the induced output current generated by that gain are determined in large part by the transit time of the carriers in the device. The initial photoelectron, injected into the multiplication region at $t=0$, drifts the entire length of the device, and assuming uniform electric-field and short-circuit device conditions, this produces a rectangular current pulse in the circuit of duration $\left(L v^{-1}\right)$ and magnitude $v q L^{-1}$, so that the area is precisely the electronic charge $q$. Using the time that the photoelectron enters the multiplication region as $t=0$, if the photoelectron creates an electron-hole pair at the first gain stage, at time $s$, the daughter electron gives rise in the circuit to a current pulse of magnitude $v q L^{-1}$ that contributes to the photocurrent for a duration of time $(J-1) s$; the conjugate hole gives rise to a similar magnitude current pulse for a duration of time $s+(L-$ $D) v^{-1}$, which includes the time to transit both the remaining gain stage and the absorption region. For each successive gain stage, $j$, each of the carriers of the electron-hole pairs created by ionization produces a charge $q$ in the circuit, which contributes to the photocurrent for durations of $(J-j) s$ and $j s+(L-$ $D) v^{-1}$ respectively.

\section{A. Measurements of SCM APD impulse response}

To analyze the temporal gain distribution of the SCM APD, measurements of the impulse response of a 10-stage SCM APDs were carried out by means of simultaneous measurementsof average gain and noise power spectral intensity. The $\left\langle M_{D C}\right\rangle$ values were obtained from I-V data collected using a computer-controlled HP $4155 \mathrm{~A}$ semiconductor parameter analyzer (SPA). The SPA applied the bias to a $190 \mathrm{~K}$ cooled SCM APD fixtured on a temperature stabilized cold post inside a windowed vacuum cryochamber. A $38 \mathrm{nW}$ 1064-nm wavelength optical signal was projected from an OZ Optics OZ-2000 stabilized fiber-coupled diode laser, through the window of the cryochamber, and onto a spot that underfilled the SCM APDs optically active area. A bias tee coupled the DC component of the diode current to the SPA, and sent the AC component to an HP 8447D high-speed preamplifier, which fed either a HP 8566B spectrum analyzer or a HP 8970B noise figure meter.

The measured SCM APD data showed gains in excess of 6000 without evidence of avalanchebreakdown.

Using a 7.5-fJ 1064-nm laser pulse from a Picoquant LDH81884 diode pumped solid state (DPSS) laser ( 45 ps width), driven with a Picoquant PDL800B, impulse response data were recorded at seven different biases, corresponding to $\left\langle M_{D C}\right\rangle=50,100,250,500,1000,1500$, and 2000, respectively. At each APD bias, a series of 360 impulse response functions were captured with an Agilent DSO80804A 8-GHz scope. The impulse response data were then parsed and the means and standard deviations of the impulse responses were calculated.

Shown in Figure $\underline{4}$ are plots of the mean impulse response functions for several $\left\langle M_{D C}\right\rangle$ bias conditions. The power spectral densities, calculated by taking the square of the FFT of the impulse response functions, are shown in Figure $\underline{5}$. The data show an SCM APD gain-bandwidth product of about $50 \mathrm{GHz}$. 


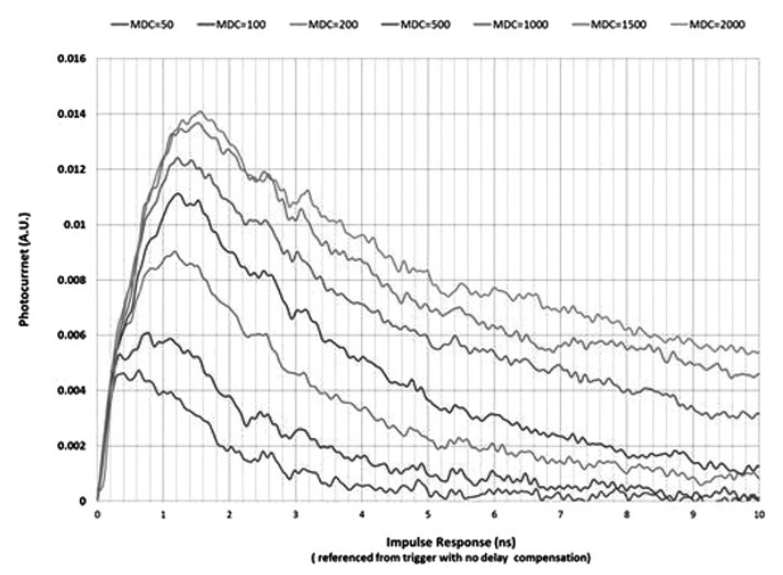

FIG. 4.Measured mean impulse response curves for various $\left\langle M_{D C}\right\rangle$ biases averaged over three-hundred and sixty 45-ps laser pulses. The times of the impulse response plotted on the $x$ axis are referenced from the trigger, without delay compensation, not from the onset of avalanche buildup.

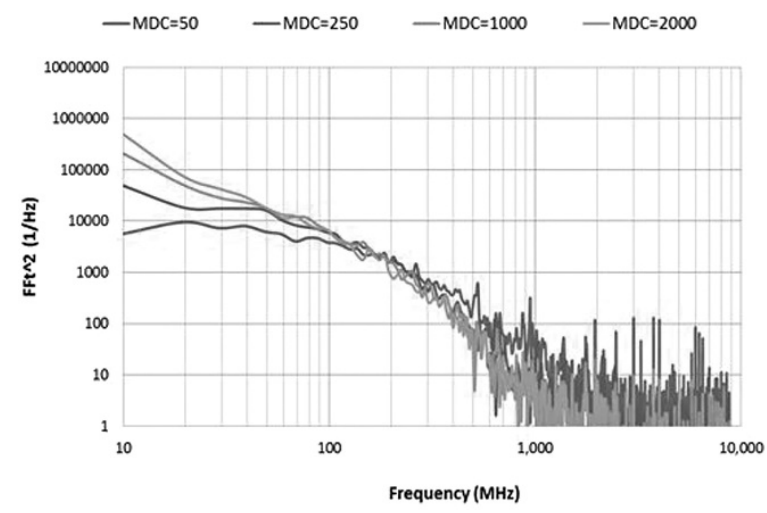

FIG. 5. The power spectral density of several of the impulse response curves calculated from the square of the FFT of the impulse response curves of Figure $\underline{4}$.

To understand the temporal dynamics of the SCM APD under each DC gain condition, the partial gains were calculated by integrating the area under the impulse response curve normalized for $\left\langle M_{D C}\right\rangle$, and the times of the impulse response that the partial gains occurred were recorded for each bias condition; these times are plotted in Figure $\underline{6}$. The data show that during the earlier times of the impulse response, the SCM APDs under high bias have a similar partial gain, whereas at later times, the times of partial gains are strongly bias dependent.

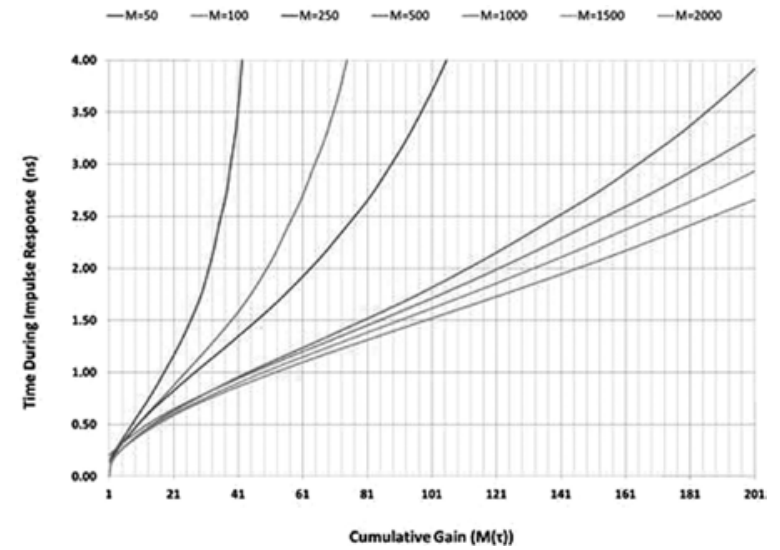

FIG. 6.Scatter plot of the time required to reach a cumulative gain (area under the impulse response function) for APDs biased for a specific DC gain. 
Figure $\underline{7}$ shows the normalized impulse response curves of Figure $\underline{4}$ plotted as a function of the estimated multiplication region transit times. The impulse response curves reveal the underlying SCM APD device structure and the bias-dependent correlations among the ionizing carriers.

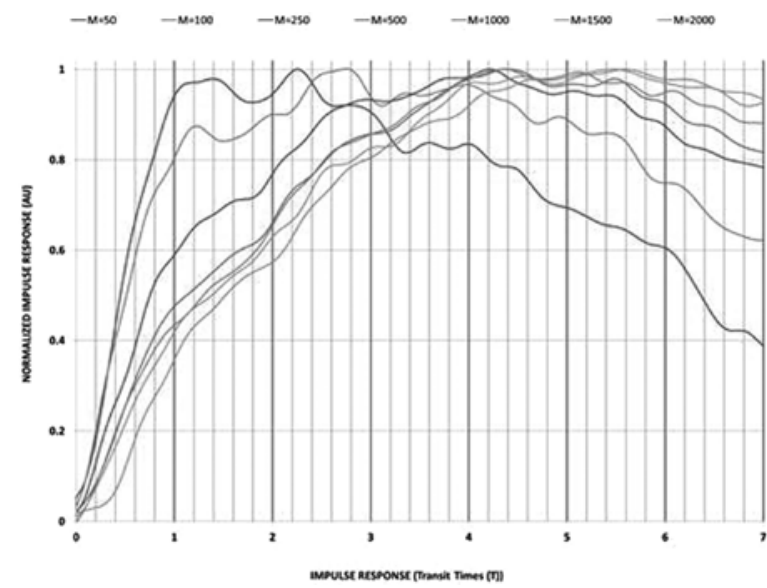

FIG. 7.Normalized impulse response curves from Figure $\underline{4}$ plotted against an estimated carrier multiplication region transit time, $\mathrm{T}=276 \mathrm{ps}$, assuming equal hole and carrier velocities.

In the SCM APD, the initial photoelectron and its primary electron progeny contribute maximally to the external current after they ionize in the 10th gain stage at about $1 T$ transit times, whereafter the electrons recombine at the cathode, causing an instantaneous drop in photocurrent. The $1 T$ transit time created holes remain in the junction and continue to contribute to the photocurrent as they travel in the $-x$ direction. This first principle generation of holes experiences its maximum instantaneous gain contribution at 1.9T transit times, when they ionize in the 1st gain stage, after having ionized, with probability $P_{h}$, or "not-ionized," with probability $\left(1-P_{h}\right)$, in each of the eight intervening gain stages. The first generation of holes continues to contribute to the photocurrent as it drifts through the absorption region until $2.75 T$ transit times, when the hole carriers recombine at the anode. At $2.8 T$ transit times, the second generation of electrons created at $1.9 T$, ionize in the 10th gain stage; they immediately recombine at the cathode, whereas their conjugate holes continue to induce photocurrent until about $4.55 T$ transit times.

From this discussion, it can be seen that the SCM APD photocurrent is induced by generational carrier contributions that are dominated by the peak numbers of electron-hole pairs created by electrons ionizing in the 10th gain stage, initially at $1 T$ transit times and subsequently at $1.8 T$ transit time increments. Ionization in the 10th gain stage causes electron and hole induced currents that extend $0 T$ and $1.75 T$ transit times, respectively. The hole ionization feedback, which peaks as the holes traverse the 1st gain stage, delayed from the electron ionization peaks by $0.9 T$ transit times, results in electron and hole induced photocurrent contributions of $0.9 T$ and $0.85 T$ transit times, respectively. It is the unique $P_{e}$ and $P_{h}$ values at each bias that contributes to the "peak" and "saddle" structure see in each impulse response curve.

After about 5.5T transit times, about the time of the maximum photocurrent contribution by the third principle wave of ionizing electrons, the branches of the ionization chains are "pruned" as the carriers leave the junction at a faster rate than new ionization branches are created. For each $\left\langle M_{D C}\right\rangle$ bias, the slopes of the exponential decays of the impulse response curves are proportional to the bias-dependent $P_{e}$ and $P_{h}$ values and by the number of gain stages. The impulse responses end after all possible ionization chains have completed and the carriers leave the junctions. 
This picture is oversimplified by its assumption of equal carrier velocities; however, the analysis of the recursive nature of the discrete gain stages is useful for understanding the time evolution of the impulse response properties. $\underline{12,16,20}$

\section{B. Statistics of an impulse response}

The process of carrier impact ionization is inherently noisy and, in addition to inducing gain fluctuations, also introduces time response fluctuations. The impulse-response can be modeled as a stochastic process composed of the statistically correlated random variables of avalanche buildup time (pulse duration) and integrated signal (the multiplication factor).

By analyzing the temporal dynamics of the ionization chains and adding the current contributions from all the offspring electrons and holes that are traveling in the multiplication region at time $t$, a buildup-time-limited impulse response values can be calculated. More precisely, if $Z_{e}(t, x)$ is the total number of electrons resulting from an initial parent electron born at location $x$ at $t$ units of time after its birth and $Z_{h}(t, x)$ is the total number of holes resulting from an initial parent electron at location $x$ at $t$ units of time after its birth, and $v_{e}$ and $v_{h}$ are the saturation velocities of the electron and hole, respectively, then assuming a single carrier injected at $x=0, \underline{(11)}$ can be expressed as

$$
\mathrm{I}(t)=\frac{q}{w}\left[v_{e} Z_{e}(t, 0)+v_{h} Z_{h}(t, 0)\right]
$$

The statistics of $I(t)$ can, therefore, be readily calculated from the joint statistics of $Z_{e}(t, 0)$ and $Z_{h}(t, 0) . \underline{17}$

A calculation of the evolution of photocurrent excess noise over time requires knowledge of the second moment of the impulse-response function at all times. To calculate the integrated photocurrent variance, the impulse response autocorrelation function is also necessary. $\underline{\underline{18,19}}$

A number of studies on the statistics of the impulse response function for conventional (continuousmultiplication) APDs have been previously conducted. The impulse response function statistics for the deadspace multiplication model, for which the ionizationsprocess has a nonlocalized nature, have also been studied. $\underline{20}$ Many of the techniques used to calculate the second-order impulse response statistics in conventional APDs require intensive computations, $\underline{\underline{21}}$ and typically involve solving coupled integral or differential equations in multiple variables. Bandyopadhyay et al. $\underline{22}$ proposed a computationally simple approach for calculating the mean impulse response function. Their theory, however, does not address the variance and the autocorrelation impulse response function. Hayat et al. .23 formulated a recurrence theory for avalanche multiplication that included the gain statistics and impulse response curve under non-uniform static electric fields. Tan et al. ${ }^{24}$ later extended that theory to accommodate for stochastic carrier velocity. Hayat and Dong ${ }^{17}$ derived an integral equation approach to calculate the avalanche duration pdf, which they termed the random-response time, and showed how to calculate its mean value and the mean of its inverse, as it relates to the APD bandwidth. Their technique was restricted to constant carrier velocities and, although it allowed for an arbitrary ionization path length pdf, these authors used the technique only to assess the avalanche duration pdf for local ionization. Sun and Hayat $\underline{25}$ subsequently generalized this approach with a probabilistic model that enables computation of the probability generating function and allows us to evaluate the joint pdf of the avalanche duration and the avalanche multiplication and to determine the statistical correlation between these two quantities. A parametric model,using the gain and the buildup time as parameters, was also developed to approximate the APD's stochastic impulse-response function. ${ }^{26}$ This allowed for computation of the APD'sstochastic impulse response moment-generating function (mgf)..$\underline{ }$ 


\section{Numerically model of SCM APD excess noise}

To describe the SCM APD's properties, we developed a model, whose framework is based on dead space multiplication theory (DSMT). $\underline{28}$ The DSMT models include an age-dependent recursive theory for avalanche multiplication that facilitates the calculation of the mean, variance, and excess noise factor of the impulse-response function. $\frac{29}{}$ To accurately modelthe temporal dynamics of the mean, variance, and excess noise characteristics of a SCM APDof the type shown in Figure $\underline{1}$, we modified the DSMT to allow for the spatial distribution of the impact ionizations for arbitrarily heterostructured gain stages.

To accommodate carrier phonon scattering in low field regions of the multiplication region (the "cool down" region of the SCM APD gain stages (i.e., region "iii" of Figure 2)), the modelfor the pdf of the distance to the first impact ionization was modified to include carrier relaxation mechanisms. Carrier relaxation was accommodated by developing "scattering aware" ionization coefficients, in which we assumed that the energy accumulated by a carrier was reset to zero once the carrier traveled a nominal distance under the influence of an electric field below a certain threshold. In our studies, we assumed that a carrier loses its energy and ceases to be able to impact ionize if it travels for $30 \mathrm{~nm}$ under an electric field below $10^{3} \mathrm{~V} / \mathrm{cm}$. These scattering aware coefficients were then used to modify the DSMT's non-localized ionization coefficients. This new extension of DSMT allowed us to compute the pdf of the distance to the first ionization event in arbitrarily structured devices and to generate the impulse response function at arbitrary times within the impulse response.

Using recursive equations, the DSMT models were used to calculate the numbers of impact ionization events triggered by electrons and holes in arbitrary sub-regions of the SCM APDsmultiplication region were determined. The ionization counts were used to characterize the joint pdf of the stochastic partial gain, $M_{D C}(\tau)$, and its stochastic avalanche duration time, $\tau$, resulting from a single avalanche trigger. The stochastic parametric model, in terms of $\left\langle M_{D C}\right\rangle, M_{D C}(\tau)$, and $\tau$, was then used to approximate the low order statistics of the SCM APD's impulse-response function.

Figure $\underline{8}$ plots the probability density (mass) function (pdf), $P(n, t, x=0)$, for a single edge injected electron, showing the probability of $n$ carrier generation at each time of the impulse response function when the SCM APD is biased for $\left\langle M_{D C}\right\rangle=937$. The $n=1$ plot illustrates a discretized drop in probability through the duration of the $1 T$ transit time, showing a nearly equal probability of the possible carrier number outcomes at $1 T$ transit times.

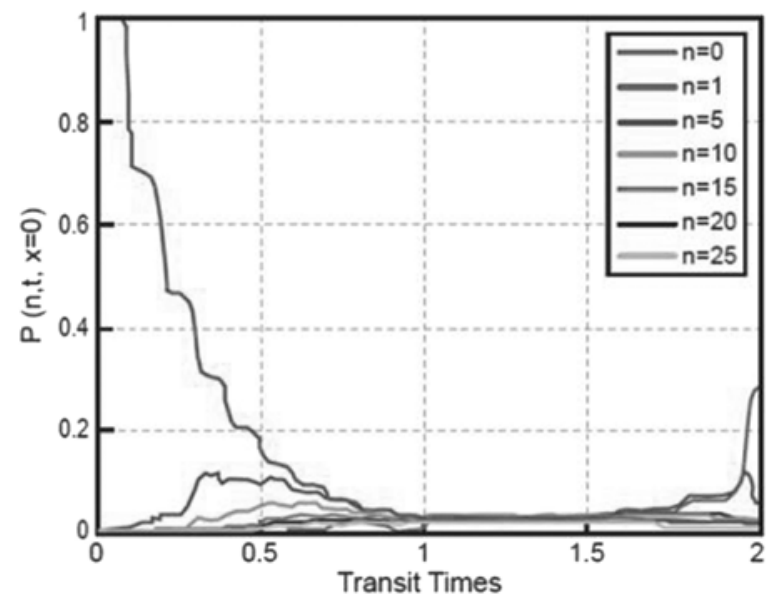

FIG. 8.Some terms of the probability density (mass) function (pdf) of the $M_{D C}=937$ bias impulse response function, at each time, $P(n, t, x=0)$ corresponding to an electron edge injection. 
The numeric model of $F\left(M_{937}\right)$ is shown in Figure $\underline{3}$ and shows good agreement with measured data, as well as the Monte Carlo simulated results and the analytical values predicted by $\left(10 ; k_{s}=0.036\right)$ for similar DC gain operation.

\section{Calculation of the mean impulse-response function}

The statistics of $I(t)$ were readily calculated from the joint spatial and temporal statistics of the electron and hole ionization events that comprise the branching ionization chains. We defined $Z_{e}(t, x)$ and $Z_{h}(t, x)$ as the total stochastic number of impact ionizations effected by electrons only and holes only, respectively, at time $t$, when the avalanche process is triggered by a parent electron at location $x$. Similarly, we defined $Y_{e}(t, x)$ and $Y_{h}(t, x)$ as the total stochastic number of impact ionizations effected by electrons only and holes only, respectively, at time $t$, when the avalanche process was triggered by a parent hole at location $x$. The mean quantities $z_{e}(t, x)=$ $\left\langle Z_{e}(t, x)\right\rangle, z_{h}(t, x)=\left\langle Z_{h}(t, x)\right\rangle$ and $y_{e}(t, x)=\left\langle Y_{e}(t, x)\right\rangle, y_{h}(t, x)=\left\langle Y_{h}(t, x)\right\rangle$ were calculated using the recursive equationsdescribed in Ref. $\underline{20}$.

We calculated the total number of electrons and holes produced by an injected electron in one equation by adding the recursive equations for $Z_{e}(t, x)$ and $Z_{h}(t, x)$ and called the sum $Z(t, x)$. With this, we recast the mean as

$$
i(t)=\langle I(t)\rangle \frac{q v}{w} z(t, 0)
$$

For the numeric modeling work described here, we simplified the problem by assuming that holes and electrons travel at approximately the same velocity, so that $v_{e}=v_{h}$, and by assuming a absorption region of negligible width (i.e., $D=L$ ). Figure $\underline{9}$ plots the simulated impulse response obtained from the probability mass function for the case of $\left\langle M_{D C}\right\rangle=26$ and $\left\langle M_{D C}\right\rangle=937$. It can be seen that, as was the case for the measured SCM APD impulse response data, a significant fraction of the device's cumulative gain, $M_{D C}(\tau)$, occurs during $T=1$ transit time and is dominated by ionization of the initial photoelectron and its progeny as they cross the stages of the multiplication region. The figure shows that, for both bias conditions, the magnitude of the response is similar during the initial transit. At $1 T$ transit times, the ratio $I_{937}(T) / I_{26}(T)$ is only 1.6 , despite their $\left\langle M_{D C}\right\rangle$ ratio of 36 .

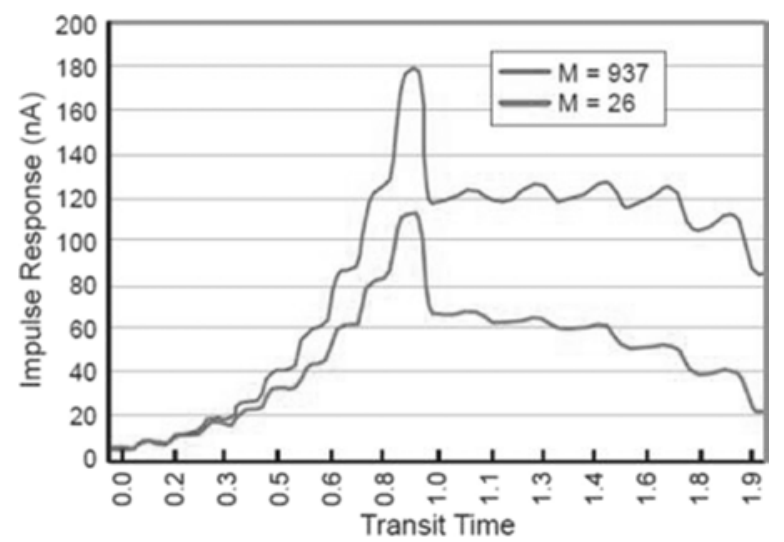

FIG. 9.Impulse responses for $M_{D C}=937$ and $M_{D C}=26$ developed by the DSMT numeric models using the timeresolved carrier distribution functions, which include scattering coefficients.

The second moment of the impulse response can be computed as 


$$
i_{t}(t)=\frac{q^{2} v^{2}}{w^{2}}\left\langle z^{2}(t, 0)\right\rangle
$$

and the standard deviation is computed as

$$
\sigma(t)=\sqrt{i_{2}(t)-\left(i_{2}(t)\right)^{2}}
$$

Figure 10 shows the numerically modeled mean impulse response, (14), and standard deviation, (15), as a function of carrier transit time over the early portion of the impulse response for an $\left\langle M_{D C}\right\rangle=937$ biased SCM APD. For optimal performance, the signal-to-noise ratio $(S N R)$ of a pulse should be large within the period of time in which most of the energy is contained. From these data, the $S N R$ can be shown to be $S N R \approx 1$, up until $2 T$ transit times, about when the holes created by the photoelectron and its progeny in 10th gain stage, at $1 T$ transit times, exit the multiplication region. Unlike the measured data, where the holes traverse a $1.5-\mu \mathrm{m}$ absorption region, the numerical model assumes no absorption region, so the hole carriers leave the junction at $2 T$ transit times. From $2 T<\tau<4 T$ transit times, the noise has another plateau with a $S N R \approx 0.5$. Taking into consideration the influence on the photocurrent by the absorption region, the $4 T$ transit period is equivalent to the 5.5T peak of the measured SCM APD impulse response data (see Figure 7 ). At all later transit times, the SNR drops due to the variation in gain that results from stronger hole ionization feedback.

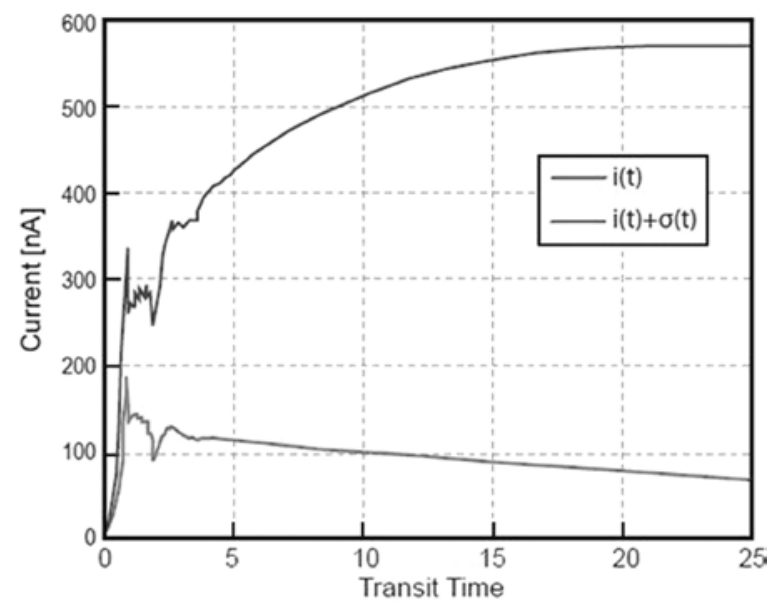

FIG. 10.Simulated impulse response, $\mathrm{i}(\mathrm{t})$ (red curve), and impulse response plus the standard deviation, $\mathrm{i}(\mathrm{t})+\sigma(\mathrm{t})$ (blue curve), as a function of the electron transit time for a gain $M_{D C}=937$ using the scattering-aware ionization coefficients.

\section{E. Calculation of the probability distribution function of the impulse response function}

We calculated the probability mass function, $P(n, t)=\operatorname{Pr}\{I(t)=(q v / w) n\}$, following the approach of Hayat and Saleh, $\underline{20}$ in which recursive equations are solved to yield the mgf of the stochastic quantities $Z(t, x)$ and $Y(t, x)$, from which the $m g f$ of $I(t)$ is derived. We obtained the probability mass function of $I(t)$ using a simple Fourier inversion applied to the mgf. The numerically modeled instantaneous excess noise, $F\left(t, M_{937}(t)\right)$, shown in Figure 11 represents, for the times of the impulse response up to $T=2$ transit times, the excess noisecharacteristics of the carriers present in the junction at each time. 


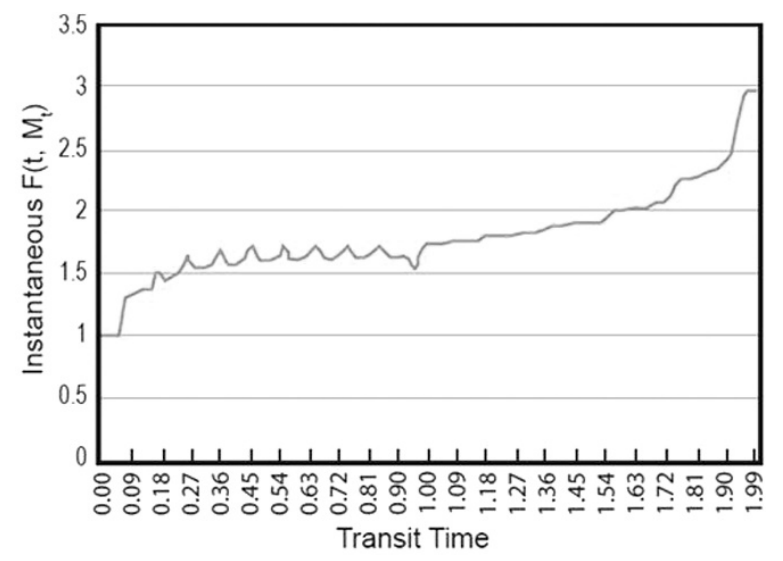

FIG. 11.Instantaneous excess noise estimated from $\left(\operatorname{var}\left(\mathrm{I}_{t}\right)+\mathrm{M}_{t}{ }^{2}\right) / \mathrm{M}_{\mathrm{t}}^{2}$ for various $\mathrm{M}_{D C}$ values.

In Figure $\underline{12}$, plotted as a function of carrier transit time, are the partial gain, $M_{937}(\tau)$ and cumulative instantaneous excess noise $F\left(M_{937}(\tau)\right)$. On the figure's secondary $y$-axis are plotted, on a linear scale, the numerically calculated impulse response, $I_{937}(t)$ data. As with the measured data of Figure $\underline{9}$, there is a first subpeak in the impulse responses that occurs at $1 T$ transit times, where the partial gain is $M_{937}(T) \approx 12$. At the second peak of the impulse response, $M_{937}(2 T) \approx 23$ and $F\left(M_{937}(2 T)\right) \approx 1.8$.

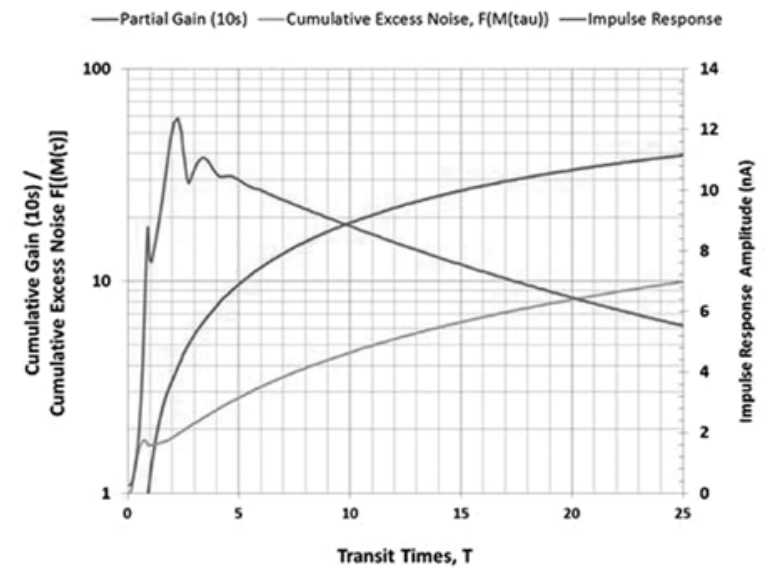

FIG. 12.Plot of the excess noise of partial gain of the impulse response for $M_{D C}=937$ bias plotted as a function of transit times. Also shown, for reference, is the numerically modeled impulse response.

\section{DISCUSSION}

At the early times of the impulse response, the numerical models of the SCM APD predict a cumulative excess noise for partial gain, $F\left(M_{D C}(\tau)\right)$ lower than the DC excess noise, $F\left(M_{D C}\right)$, for an APD biased for an equivalent gain. For example, when $M_{937}(\tau)=100$ (at $5 T$ transit times), the cumulative instantaneous excess noise is 2.8 , but when $M_{D C}=100$, the DC excess noise is 5.6 (see Figure $\underline{3}$ ).

The data support the notion of a multimodal gain process, which is dominated during the early times (i.e., $\tau$ $<2 T$ transit times) of the impulse response by the partial gain contributions of the photoelectron and its progeny, both holes and electrons, created during $1 T$ transit times. During these times, the partial gain can be approximated by the discrete multi-gain stage APD models of (7), with a $P_{e}$ characteristic of the $\left\langle M_{D C}\right\rangle$ bias condition, according to $(7) P_{e}=M_{D C}(\tau)^{1 / s}-1$.

Using $k_{s}=0.036$ used above to fit (10) to the experimental data, from (9) we can estimate that to achieve a DC gain of 937 a $P_{e}=0.369$ is required. Assuming negligible hole ionizationfeedback, using these $P_{e}$ values during the preliminary transit time, we can anticipate during $1 T$ transit times, a total average carrier gain of $(1+$ 
$0.369)^{10} \approx 23.12$ for $\left\langle M_{D C}\right\rangle=937$. This is consistent with the numerically modeled data (Figure $\underline{12}$ ) and the measured data (Figure 6 ). At the lowest bias, $\left\langle M_{D C}\right\rangle=50$, the measured data show that at $M_{50}(\tau) \approx 12$, which, from (7)yields $P_{e}=0.275$. At later times of the impulse response, hole ionization feedback must be considered.

In Figure $\underline{13}$ are plotted the numerically modeled $F\left(M_{937}\right)$ and $F\left(M_{937}(\tau)\right)$ data, along with plots of (10) parameterized by $P_{e}=0.37$ with both $k_{s}=0.0350$ and $k_{s}=0$. Another plot of (10), parameterized by $P_{e}=0.37$ is shown for $k_{s}=0.012$ for $F\left(M_{937}\right)<100$ and $k_{s}=0.015$ for $100<F\left(M_{937}\right)<300$. At partial gains less than about 10 , the numerically modeled $F\left(M_{937}(\tau)\right)$ data are slightly above that modeled by $(10)$. This is not surprising, as unlike the analytical DC models, the numeric models include the stochastic random variables of both avalanche buildup time and multiplication. The DSMT generated $P(n, t, x=0)$ plots (Figure $\underline{8}$ ) illustrate the temporal variability of the avalanche buildup process. The $n=1$ curve shows a wide range of probabilities over the first transit period. The wide range of $n=1$ probabilities during the initial transit time is a result of the chance of failure of the photoelectron to multiply earlier in the chain of gain stages. The plot of $\left(10 ; k_{s}=0.012\right)$ begins to approximate the numerically modeled partial gain data after $M_{937}(\tau)=10$, which, from Figure $\underline{8}$, probably occurs at about $0.5 T$ transit times.

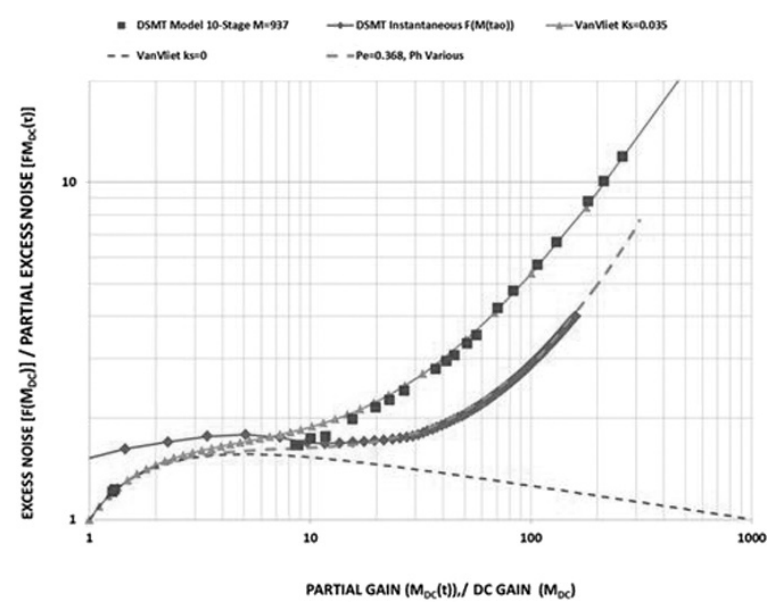

FIG. 13.Plots of the numerically modeled excess noise of $D C$ gain $F\left(M_{D C}\right)$ and the cumulative excess noise of partial gain $\mathrm{F}\left(\mathrm{M}_{\mathrm{\tau}}\right)$ for a $\left\langle\mathrm{M}_{\mathrm{DC}}\right\rangle=937$ bias, compared to the analytic models of $(10)$ using $\mathrm{J}=10$ gain stages and $\left(P_{e}=0.037, k_{s}=0.035\right),\left(P_{e}=0.037, k_{s}=0\right)$, and $\left(P_{e}=0.037, k_{s}=0.012\right)$.

Another difference between the numerically modeled cumulative instantaneous excess noiseand the DC excess noise models of $\underline{(10)}$ is that the numeric models have a $P_{e}$, fixed by the bias, at all times, whereas $\underline{(10)}$ has a different $P_{e}$ value at each gain as calculated from $(9)$. Using $\left(9 ; \mathrm{k}_{\mathrm{s}}=0.035\right)$ it can be shown that in order to achieve $\left\langle M_{D C}\right\rangle=937$, a $P_{e}=0.369$ and $P_{h}=0.013$ are required. By examining the point in Figure $\underline{8}$ where $P(1,0.5 T, 0)$ is approximately equal to $P(5,0.5 T, 0)$, which occurs after the photoelectron ionizes, or not, in the 5th gain stage, and the values of $P(1,1 T, 0)$ and $P(25,1 T, 0)$, after the time the photoelectron transits the 10th gain stage, using (7) we can confirm our estimate of $P_{e} \approx 0.37$ at the early times of the impulse response.

The $P(0,2 T, 0)$ value of $\sim 0.3$ is indicative of a $P_{h}$ nearly 0 over most of the first two transit times; it is apparent, over this time period, that carriers are leaving the junction at a rate much faster than they are being created. From the fit of the numeric cumulative instantaneous noise data with $\left(10 ; k_{s}=0.012\right)$ at a partial gain of 100 at

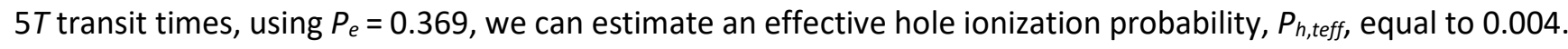
This is about $1 / 3$ rd the hole ionization probability estimated for DC gains of comparable magnitude to the partial gain. It is not until later times, that $k_{s}=0.035$, resulting in $P_{h}=0.013$. 


\section{SUMMARY AND CONCLUSION}

The work presented demonstrates a new method of predicting APD photoreceiver performance and the data support a conclusion that commonly used pseudo-DC APD noisemodels are inadequate for describing the gain and noise fluctuations of the circuits used in pulse detection, wherein the pulses are shorter than the avalanche buildup time. To the best of our knowledge, this is the first application of numeric models descriptive of measuredtime-resolved APD impulse responses used for predicting the instantaneous gain and noiseproperties of the photocurrents in threshold decision circuits.

A numeric DSMT model, modified to take into account phonon scattering in low field regions, was used to model the SCM APDs gain and noise properties over a range of biases. After the model was shown to approximate measured DC excess noise data and Monte Carlo simulations, the instantaneous and partial gain and noise properties of the SCM APD were modeled. Unlike the analytic models, the DSMT model does not involve any fitting parameters (i.e., $k$ or $k_{s}$ ) to the empirical data; it only uses universal parameters for the nonlocalized ionization coefficients and the materials' ionization threshold energies.

The numerical models were used to gain insight into the SCM APD carrier dynamics over the times of the impulse response under different bias conditions. It was confirmed that during the early impulse response times, instantaneous gains are higher and the instantaneous excess noise lower than at the later times of the impulse response. Under $M_{D C}=937$ bias, the numeric models show that at a partial gain of 100 , the effective excess noise of the SCM APDis less than 3 . This is about $50 \%$ the numerically modeled pseudo-DC excess noise, which was shown to be in good agreement with measured data and with Monte Carlo models.

The plots of $\left(10 ; k_{s}=0.035\right)$ showed excellent correspondence with the DSMT modeled and measured DC gain and excess noise data. This finding confirms previous work that predicted that a single-carrier multiplication APD with dead space can be approximated by a superlattice APD for which the separation between the layers is the dead space and the ionization probability per layer is obtained by matching the gains of the two devices. $\frac{30}{}$ With the data from the DSMT model, we were able to estimate, at the longer times of the impulse response, an electron ionization probability of about $P_{e}=0.368$ and a hole ionizationprobability of $P_{h}=0.013\left(k_{s}=0.035\right)$. These values were then used to parameterize the Van Vliet $(10)$ and Mclntyre (6) models. The McIntyre models were shown to apply only at high DC gain levels, and were not useful for predicting the instantaneous noise properties. At a partial gain of 100, the numerically modeled cumulative instantaneous excess noise of the SCM APD is less than $50 \%$ that predicted by $(6)$ for a $M_{D C}$ equal to the partial gain. Significantly, the numerically modeled SCM APD data are $7 \%$ the excess noise predicted by the McIntyre models for common bulk InP multiplication layer APDs calculated using $(6 ; \mathrm{k}=0.4)$.

A fit of $(10)$ using the estimated $P_{e}=0.368$ values, and $k_{s}=0.012$ provided a reasonable estimation of the cumulative excess noise obtained at the partial gain obtained at early times of the impulse response. When $\mathrm{k}_{\mathrm{s}}$ was varied as a function of gain, to reflect the temporal dynamics of the carrier populations gain and noise statistics, the model could be fitted to the numeric model over all times. However, it is important to note, again, that $(10)$ was developed to describe DC operating conditions; its usefulness in describing the

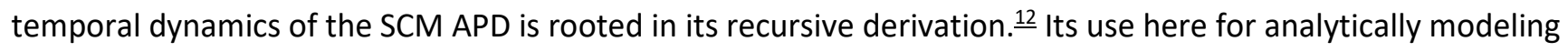
the cumulative instantaneous excess noise using parameters based on device properties has not been proven. While it may be possible to modify $(9)$ and $(10)$ so that they incorporate the temporal correlations of the carriers over the partial gains obtained during the SCM APDs impulse response, we do not offer proof, and we leave that work for future efforts. Future work will also include using the predictive models to predict measuredreceiver operating characteristic (ROC) curves, and repeating the work presented here using data from 14 stage SCM APDs. 


\section{REFERENCES}

1.A. van der Ziel, Noise in Measurements (John Wiley \& Sons, 1976).

2.R. E. Burgess, " Homophase and heterophase fluctuations in semiconducting crystals," Discuss. Faraday Soc. 28, 151-158(1959). https://doi.org/10.1039/df9592800151,

3.H. Kühn and D. G. Welsch, "Transient-gain-assisted noise reduction in photodetection of nonclassical light," Phys. Rev. Lett. 67(5), 580-583 (1991). https://doi.org/10.1103/PhysRevLett.67.580,

4.R. J. Mclntyre, "Multiplication noise in uniform avalanche diodes," IEEE Trans. Electron Devices ED13, 164168 (1966). https://doi.org/10.1109/T-ED.1966.15651,

5.J. N. Hollenhorst, “ A theory of multiplication noise," IEEE Trans. Electron Devices 37, 781788 (1990). https://doi.org/10.1109/16.47786,

6.W. A. Lukaszek, A. van der Ziel, and E. R. Chenette, Solid-State Electron. 19, 57

(1976). https://doi.org/10.1016/0038-1101(76)90134-9,

7.K. M. van Vliet and L. M. Rucker, "Theory of carrier multiplication and noise in avalanche devices-Part I: Onecarrier processes," IEEE Trans. Electron Devices ED-26, 746-751 (1979). https://doi.org/10.1109/TED.1979.19489,

8.F. Capasso, W. T. Tsang, and G. F. Williams, "Staircase solid-state photomultipliers and avalanche photodiodes with enhanced ionization rates ratio," IEEE Trans. Electron Devices ED-30, 381390 (1983). https://doi.org/10.1109/T-ED.1983.21132,

9.F. Capasso, " Avalanche photodiodes with enhanced ionization rates ratio: Towards a solid state photomultiplier," IEEE Trans. Nucl. Sci. 30(1), 424428 (1983). https://doi.org/10.1109/TNS.1983.4332303,

10.A. Van Der Ziel, Y. J. Yu, G. Bosman, and C. M. Van Vliet, " Two simple proofs of Capasso's excess noise factor FN of an ideal N-stage staircase multiplier," IEEE Trans. Electron Devices ED-33(11), 18161817 (1986). https://doi.org/10.1109/T-ED.1986.22746,

11.K. M. van Vliet, A. Friedmann, and L. M. Rucker, "Theory of carrier multiplication and noise in avalanche devices - Part II: Two carrier processes," IEEE Trans. Electron Devices ED-26, 752764 (1979). https://doi.org/10.1109/T-ED.1979.19490,

12.M. C. Teich, K. Matsuo, and B. E. A. Saleh, "Excess noise factors for conventional and superlattice avalanche photodiodes and photomultiplier tubes," IEEE J. Quantum Electron. 22(8), 11841193 (1986). https://doi.org/10.1109/JQE.1986.1073137,

13.K. Matsuo, M. C. Teich, and B. E. A. Saleh, "Noise properties and time response of the staircase avalanche photodiode," IEEE Trans. Electron Devices 32(12 ), 2615-2623 (1985). https://doi.org/10.1109/TED.1985.22392,

14.S. Ramo, "Currents induced by electron motion," Proc. IRE 27(9) ), 584585 (1939). https://doi.org/10.1109/JRPROC.1939.228757,

15.K. F. Brennan, Y. Wang, M. C. Teich, B. E. A. Saleh, and T. Khorsandi, "Theory of temporal response of a simple multiquantum-well avalanche photodiode," IEEE Trans. Electron Devices 35(9), 14561467 (1988). https://doi.org/10.1109/16.2578,

16.K. M. Van Vliet, A. Friedmann, and L. M. Rucker, "Theory of carrier multiplication and noise in avalanche devices - Part I: Two carrier processes," IEEE Trans. Electron Devices ED-26, 752-764(1979).

17.M. M. Hayat and G. Dong, "A new approach for computing the bandwidth statistics of avalanche photodiodes," IEEE Trans. Electron Devices 47, 1273-1279 (2000). https://doi.org/10.1109/16.842973,

18.M. M. Hayat, B. E. A. Saleh, and J. A. Gubner, "Bit-error rates for optical receivers using avalanche photodiodes with dead space," IEEE Trans. Commun. 43(1), 99-

106 (1995). https://doi.org/10.1109/26.385938,

19.M. M. Hayat, O. Kwon, Y. Pan, P. Sotirelis, J. C. Campbell, B. E. A. Saleh, and M. C. Teich, " Gain-bandwidth characteristics of thin avalanche photodiodes," IEEE Trans. Electron Devices 49(5), 770781 (2002). https://doi.org/10.1109/16.998583, 
20.M. M. Hayat and B. E. A. Saleh, "Statistical properties of the impulse response function of double-carrier multiplication avalanche photodiodes including the effect of dead space," J. Lightwave Technol. 10, 1415-1425 (1992). https://doi.org/10.1109/50.166785,

21.G. Kahraman, B. E. A. Saleh, and M. C. Teich, "Spectral properties of photocurrent fluctuations in avalanche photodiodes," J. Lightwave Technol. 10(4 ), 458-467 (1992). https://doi.org/10.1109/50.134199,

22.A. Bandyopadhyay, M. J. Deen, L. E. Tarof, and W. Clark, " A simplified approach to time-domain modeling of avalanche photodiodes," IEEE J. Quantum Electron. 34, 691699 (1998). https://doi.org/10.1109/3.663452,

23.M. M. Hayat, B. E. A. Saleh, and M. C. Teich, "Effect of dead space on gain and noise of double-carrier multiplication avalanche photodiodes," IEEE Trans. Electron Devices 39(3), 546552 (1992). https://doi.org/10.1109/16.123476,

24.C. H. Tan, J. P. R. David, S. A. Plimmer, G. J. Rees, R. C. Tozer, and R. Grey, " Low multiplication noise thin Al Ga As avalanche photodiodes," IEEE Trans. Electron Devices 48(7), 13101317(2001). https://doi.org/10.1109/16.930644,

25.P. Sun and M. M. Hayat, " A linear equalizer for high-speed APD-based integrate-and-dump receivers," IEEE Commun. Lett. 9, 1073-1075 (2005). https://doi.org/10.1109/LCOMM.2005.1576593,

26.P. Sun, M. M. Hayat, B. E. A. Saleh, and M. C. Teich, " Statistical correlation of gain and buildup time in APDS and its effects on receiver performance," IEEE J. Lightwave Technol. 24, 755768(2006). https://doi.org/10.1109/JLT.2005.862436,

27.P. Sun, M. M. Hayat, and A. K. Das, "Bit error rates for ultrafast APD based optical receivers: Exact and large deviation based asymptotic approaches," IEEE Trans. Commun. 57(9), 27632770(2009). https://doi.org/10.1109/TCOMM.2009.09.080056,

28.M. A. Saleh, M. M. Hayat, B. E. A. Saleh, and M. C. Teich, " Dead-space-based theory correctly predicts excess noise factor for thin GaAs and AIGaAs avalanche photodiodes," IEEE Trans. Electron Devices 47(3 ), 625633 (2000). https://doi.org/10.1109/16.824739,

29.2010 IEEE Photonics Society Annual Meeting. STC.UNM Provisional patent pending. US patent application $61 / 456,455$

30.B. E. A. Saleh, M. M. Hayat, and M. C. Teich, "Effect of dead space on the excess noise factor and time response of avalanche photodiodes," IEEE Trans. Electron Devices 37(9 ), 19761984(1990). https://doi.org/10.1109/16.57159, 